\title{
Metagenomic and metabolomic analyses reveal distinct stage- specific phenotypes of the gut microbiota in colorectal cancer
}

\author{
Takuji Yamada ${ }^{1 *}$
}

${ }^{1}$ School of Life Science and Technology, Tokyo Institute of Technology 2-12-1 Ookayama, Meguro-ku, Tokyo, 152-8550, JAPAN

\begin{abstract}
Colorectal cancer (CRC) worldwide affects over a quarter of a million people each year. Most sporadic CRCs develop through formation of polypoid adenomas and are preceded by intramucosal carcinoma (Stage 0), which can progress into malignant forms. Detection of early cancers and their endoscopic removal are priorities for cancer control. Human gut microbiome has been associated to CRC development, and its comprehensive characterization is of a great importance to assess its potential as a diagnostic marker. We performed whole shotgun metagenomic sequencing and CE-TOFMS-based metabolomic studies on fecal samples collected from 616 participants undergoing colonoscopy to assess taxonomic and functional characteristics of gut microbiota and metabolites. As a result, microbiome and metabolite shifts were apparent in cases of multiple polypoid adenomas (MP) and Stage 0, in addition to more advanced lesions (Stage I/II and Stage III/IV). Two distinct patterns of microbiome elevations were found $(\mathrm{P}<0.005)$. First, $\mathrm{CRC}$-associated species including Fusobacterium nucleatum were elevated continuously from Stage 0 to more advanced stages. Second, Atopobium parvulum and Actinomyces odontolyticus, which co-occurred in Stage 0, were elevated only in MP and/or in Stage 0. Metabolome analyses showed elevation of branched-chain amino acids and phenylalanine in Stage 0 and bile acids including deoxycholate in MP and/or Stage 0. Metagenomic functional analyses showed amino acid metabolism and sulfide producing pathways were associated with CRCs. Our study indicates possible etiological and diagnostic significance of fecal microbiota and metabolite in the very early stages of CRC

(Yachida et al., Nature Medicine 2019).
\end{abstract}

* Corresponding author: takuji@bio.titech.ac.jp 